\title{
PULSED SINGLE FREQUENCY FIBER LASERS
}

\author{
Shibin Jiang \\ AdValue Photonics Inc \\ 3440 E. Britannia Drive, Suite 190, Tucson, AZ 85706, USA \\ sjiang@advaluephotonics.com
}

\begin{abstract}
Pulsed single frequency fiber lasers with $\mathrm{mJ}$ level near 1 micron, 1.55 micron and 2 micron wavelengths were demonstrated by using our proprietary highly doped fibers. These fiber lasers exhibit excellent long term stable operation with $\mathrm{M}^{2}<1.2$.
\end{abstract}

\section{INTRODUCTION}

Coherent lidar and atmospheric sensing require compact coherent pulsed narrow linewidth single frequency laser sources. High pulse energy and high peak power is preferred, especially for airborne and space-borne applications. In order to achieve high pulse energy and high peak power, free-space solid-state lasers are typically used in order to avoid the optical nonlinearity. Free-space solid-state solid state lasers are challenging for packaging. All-fiber monolithic laser sources are highly desirable for these applications, especially for airborne and space borne applications, because fiber laser sources offer a much more compact and robust solution. With the advancement of fiber lasers in the recent years fiber based component become readily available. Single-frequency pulse radiation can simply be generated from intensitymodulated distributed feedback (DFB) semiconductor laser and/or single-frequency fiber oscillators in combination with fiber based acoustic optic modulator (AOM). The pulse energy can be increased by following fiber amplifiers. However, the pulse energy and peak power of the fiber amplifiers are limited because of the stimulated Brillouin scattering (SBS). The pulse energy is generally limited to near $0.1 \mathrm{~mJ}$ level for most commercially available fiber lasers although much higher values were reported by using large mode-area fibers and photonic crystal fibers. In this paper we report single frequency pulsed fiber laser with $\mathrm{mJ}$ level near 1 micron, 1.55 micron and 2 micron laser wavelengths.

\section{RARE-EARTH DOPED GLASSES AND FIBERS}

We developed multi-component non-silica glasses as the host of the rare-earth ions, which permit a higher doping concentration because of their lessdefined glass network as compared to silica glass. $\mathrm{Yb}, \mathrm{Er}, \mathrm{Er} / \mathrm{Yb}, \mathrm{Tm}$, and $\mathrm{Tm} / \mathrm{Ho}$ doped glasses were fabricated as the core glasses using high purity chemicals with less than $1 \mathrm{ppm}$ of iron and copper. Glass melting was processed in platinum crucibles. The hydroxyl ion $\mathrm{OH}-$ concentration in these glasses are controlled in order to eliminate the non-radiative energy transfer from the excited level of rare-earth ions to $\mathrm{OH}-$. We carefully control the glass melting process in order to prevent other contaminations from ceramic insulation materials and heating elements, ensuring a low propagation loss. The absorption spectra are recorded on a UV-Vis-Nir Spectrophotometer. Refractive index of core glasses at $632 \mathrm{~nm}, 980 \mathrm{~nm}$ and $1550 \mathrm{~nm}$ are characterized. The glass transition temperature, softening temperature, and thermal expansions coefficient are measured.

Undoped glasses for the inner and external cladding layers are designed and fabricated. Refractive index of undoped cladding glasses are characterized for the calculation of the numerical aperture (NA).

Rod-in-tube fiber drawing technique is used for our fiber fabrication. The core glass rod will be drilled from a bulk rare-earth doped glasses, and the barrel of the rod will be polished to a high surface quality. Cladding glass tubes will be fabricated from undoped glasses. Both inside and 
outside surfaces of the glass tubes made from the bulk cladding glasses are polished to a high surface quality. The inside diameter of the inner cladding tube should be perfectly matched with the diameter of the core glass. The outside diameter of the inner cladding tube should be perfectly matched with the inside diameter of the outer cladding tube. Fiber drawing process is carried our in-house using a fiber drawing tower that is optimized for non-silica fiber drawing.

The high doping concentration of our non-silica fibers enables high pump absorption and high gain per unit length, which is particularly essential for high pulse energy single frequency laser amplification. We used these fibers demonstrated single frequency fiber laser, Q-switched all-fiber fiber lasers, as well as mode-locked fiber lasers.

\section{RESULTS}

We directly modulate DFB single frequency diode lasers for a few nanoseconds pulse generation and AOM modulation for a few hundred nanoseconds pulse generation. When the pulse width is relatively long, pulse shaping is necessary in order to generate Gaussian pulse after the amplification. Single-frequency pulse radiation can simply be generated from intensity-modulated distributed feedback (DFB) semiconductor laser and/or single-frequency fiber oscillators in combination with fiber based acoustic optic modulator (AOM).

For $1030 \mathrm{~nm}$ fiber laser, pulse energy of $1 \mathrm{~mJ}$ and pulse width of $200 \mathrm{~ns}$ to $300 \mathrm{~ns}$ was demonstrated. When the pulse width is $4.5 \mathrm{~ns}$, a peak power of $88 \mathrm{~kW}$ was demonstrated.

A $1550 \mathrm{~nm}$ single frequency fiber laser with an average power of $8.4 \mathrm{~W}$, pulse energy of $0.42 \mathrm{~mJ}$ and peak power of $2.1 \mathrm{~kW}(200 \mathrm{~ns}, 20 \mathrm{kHz})$ were successfully demonstrated by using an $50 \mathrm{~cm}$ long fiber. An Er fiber laser at $1532 \mathrm{~nm}$ was built as the pump source in order to improve the efficiency. A single-frequency DFB diode laser was used as the seed laser. Three-stage low-noise fiber preamplifiers were used to boost the power of the seed pulses. Narrow-band filter is used to filter ASE before it is launched into the last stage amplifier. The slope efficiency is measured to be greater than $56 \%$.
A single frequency fiber laser at $1950 \mathrm{~nm}$ was built and AOM was used to modulate the pulse. Pulse shaping was applied in order to generate Gaussian like pulse after the amplification. A Tm-doped fiber with a length of $30 \mathrm{~cm}$ was used for amplification. The pump laser is multi-mode $793 \mathrm{~nm}$ laser diode. With $45 \mathrm{~W}$ pump laser an output of near $16 \mathrm{~W}$ was demonstrated. The pulse energy is greater than $0.3 \mathrm{~mJ}$.

\section{CONCLUSIONS}

We demonstrated and commercialized single frequency pulsed fiber lasers with $\mathrm{mJ}$ level near 1 micron, 1.55 micron and 2 micron wavelengths by using our proprietary highly doped single mode fibers. Since these fibers are truly singe mode fiber, these fiber lasers exhibit stable long term operation with excellent beam quality. We are in the processing of further scale up the pulse energy. 